\title{
Partial Discharge Process and Characteristics of Oil-Paper Insulation under Pulsating DC Voltage
}

\author{
Lianwei Bao*, Jian Li ${ }^{\dagger}$, Jing Zhang*, Tianyan Jiang** and Xudong Li*
}

\begin{abstract}
Oil-paper insulation of valve-side windings in converter transformers withstand electrical stresses combining with $\mathrm{AC}, \mathrm{DC}$ and strong harmonic components. This paper presents the physical mechanisms and experimental researches on partial discharge (PD) of oil-paper insulation at pulsating DC voltage. Theoretical analysis showed that the phase-resolved distributions of PDs generated from different insulated models varied as the increase of the applied voltages following a certain rule. Four artificial insulation defect models were designed to generate PD signals at pulsating DC voltages. Theoretical statements and experimental results show that the PD pulses first appear at the maximum value of the applied pulsating DC voltage, and the resolved PD phase distribution became wider as the applied voltage increased. The PD phase-resolved distributions generated from the different discharge models are also different in the phase-resolved distributions and development progress. It implies that the theoretical analysis is suitable for interpretation of $\mathrm{PD}$ at pulsating DC voltage.
\end{abstract}

Keywords: Convert transformer, Partial discharge property, Oil-paper insulation, Pulsating DC voltage, Discharge procedure

\section{Introduction}

Converter transformers play an important role in high voltage direct current (HVDC) transmission systems [1-2]. Oil- paper insulation is one of the major types of insulation in converter transformers. Valve-side windings of HVDC converter transformers were subjected to pulsating DC voltage stresses consisting of $\mathrm{AC}, \mathrm{DC}$ and strong harmonic components [3-5]. With the increase of the transmission voltage rating, partial discharges (PDs) will happen in the weak insulation of converter transformer. It is harmful to normal operation of the converter transformer, even the whole HVDC transmission system. PD properties of oil-paper insulation under pulsating DC voltage are complicated but very important to the normal operation of the converter transformer.

Many works have been focused on detection, properties and pattern recognition of $\mathrm{PD}$ under individual voltage of AC, DC, and harmonic. Literature [4] proposed modeling of PD development in electrical tree channels under AC voltages, and some PD characterizations of streamers were proposed in publication [5]. Furthermore, some evaluations of PD patterns and oil-paper aging were presented in [6-7]. Researches about pattern recognition for PD under AC voltage were studied in publication [8-

$\dagger$ Corresponding Author: State Key Laboratory of Power Transmission Equipment and System Security and New Technology, Chongqing University, China. (jianli@cqu.edu.cn)

* State Key Laboratory of Power Transmission Equipment and System Security and New Technology, Chongqing University, China (baolianwei@cqu.edu.cn)

** College of AppliedScience and Technology, Chongqing University of Technology, China.

Received: March 25, 2015; Accepted: October 6, 2015
9]. PDs of oil-paper insulation under DC voltage were different from that under AC voltage. U. Fromm gave an overview on PDs under DC voltage and proposed a new histogram for interpretation of PD under DC voltage [10]. Publication [11] proposed the mechanism, detection and analysis of PD under DC voltage, while classification for them was presented in [12]. Besides, several new detected approaches of ultra-high-frequency (UHF) were proposed in literatures [13-14]. In addition, researches about PD properties under impulse voltages were proposed in literatures [15-17]. When it comes to HVDC converter transformers, the insulation subjected to pulsating DC voltage, which made the electric field distribution in insulation much more complex. Many research achievements about PD characteristics at AC or DC voltage cannot be directly applied to that at pulsating DC voltage. A motivation is thus given to investigate the PD process and characteristics of oil paper insulation when subjected to pulsating DC voltage.

For better interpretation of PD properties in converter transformer, this paper presents the theoretical mechanism and experimental research on PD properties of oil-paper insulation under pulsating DC voltage. First of all, pulsating DC voltages across winding-to-ground insulation of the three single-phase units of converter transformer were analyzed. The voltages consisting of $\mathrm{AC}$ and $\mathrm{DC}$ voltages with equivalent amplitudes were selected for investigation. Theoretical mechanisms were proposed for interpretation of PD properties of four insulation defect models. Furthermore, four artificial insulation defect models were designed to generate PD signals under pulsating DC voltage, which were detected by a Rogowski 
coil sensor in experiments. Experimental results showed that the phase-resolved distributions of PDs generated from the four models were in agreement with the theoretical analysis but different from each other. It was conducive for faults diagnosis based on PD signals in converter transformers.

\section{Voltages Analysis in Converter Transformer}

Fig. 1 shows the simulated result of the output voltages of the converter transformer by software PSIM. It confirms that valve-side windings of HVDC converter transformers subjected to pulsating DC voltage stresses consisting of $\mathrm{AC}$, $\mathrm{DC}$, and strong harmonic voltage components.

The ratio of $\mathrm{AC}$ peak voltage to $\mathrm{DC}$ voltage level in wye-connection unit and delta-connection unit are 1:3 and $1: 1$, respectively. The voltages across winding-to-ground insulation of the three single-phase units of transformers can be expressed as Eq. (1).

$$
u_{a d}=u_{a c} \sin (\omega t+\varphi)+u_{d c}
$$

The pulsating DC voltage with lower RF will cause more serious damages to oil-paper insulation at room temperature, where RF is defined as the ratio of AC peak voltage to DC voltage level. Thus the paper only presents the PD properties under pulsating DC voltage with $\mathrm{RF}=1$.

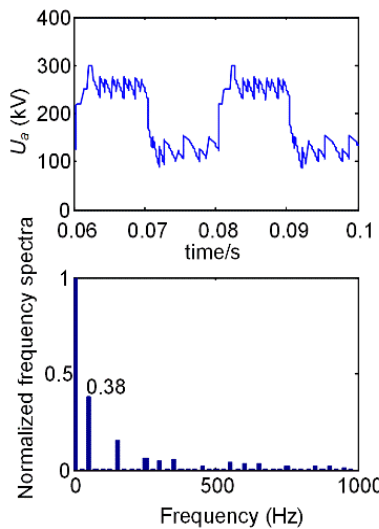

(a)

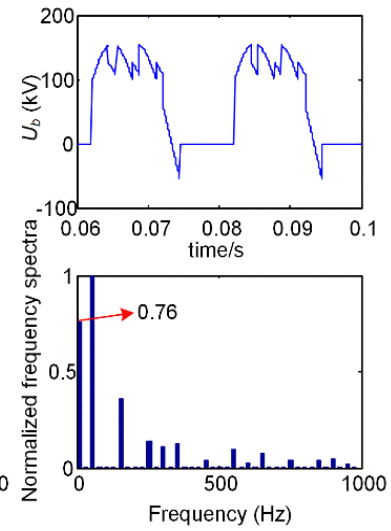

(b)
Fig. 1. Waveform of converter transformer valve-side windings: (a) Y connection; (b) $\Delta$ connection

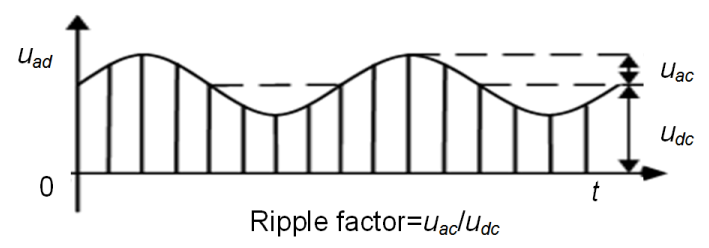

Fig. 2. Waveform combined with $A C$ and DC voltages
Table 1. List of symbols.

\begin{tabular}{c|l}
\hline Parameters & \multicolumn{1}{c}{ Descriptions } \\
\hline$u_{0}$ & $\begin{array}{l}\text { Voltage due to the applied voltage, } u_{0}=f \cdot u_{a d}, \text { where } f \text { is } \\
\text { a constant }\end{array}$ \\
\hline$u_{q}$ & Voltage due to the surface charge \\
\hline$u_{c}$ & Voltage over the cavity \\
\hline$u_{C B}$ & Breakdown voltage of the cavity \\
\hline$u_{p}$ & Impulse voltage of partial discharges \\
\hline$u_{r}$ & Residual voltage \\
\hline
\end{tabular}

\section{Physical Mechanisms of PD under Pulsating DC Voltage}

Before analysis of PD mechanisms under pulsating DC voltage, several parameters are presented in Table 1. The physical mechanisms of four insulation defects involving cavity discharge, surface discharge, floating discharge and corona discharge are shown as follows.

\subsection{Analyses of cavity discharge}

The process of cavity discharge under pulsating DC voltage is more complex than that under individual $\mathrm{AC}$ or DC voltage. PDs in gaseous cavities inside a dielectric are usually considered the most dangerous [11]. Two conditions must be fulfilled in order to start a PD. First, the magnitude of the electric field in the cavity should be higher than a minimum breakdown voltage. Second, a free electron is necessary to start the ionization process. In the PD process, the charge variation can be reflected by the electrodes between the cavity models. The PD process of cavity discharge can be depicted by a planeparallel capacitor, which is shown in Fig. 3.

When the applied voltage reaches the breakdown electrical field of the cavity, as called $E_{\mathrm{CB}}$, PD will happen in the gaseous cavity. The gas molecules will ionize to electriferous particles involving electrons and positive ions. When the external voltage is applied, the positiveions is pulled in the direction of the applied voltage, while electrons are pulled in the opposite direction. The internal surface charges in turn produce opposite voltages compared with the external voltage, which is shown in Fig. 3.

Once the maximum value of $u_{0}$ is bigger than $u_{C B}$, PD will occur in the cavity. The voltage over the cavity $u_{\mathrm{c}}$ decreases sharply due to charge accumulation on the cavity

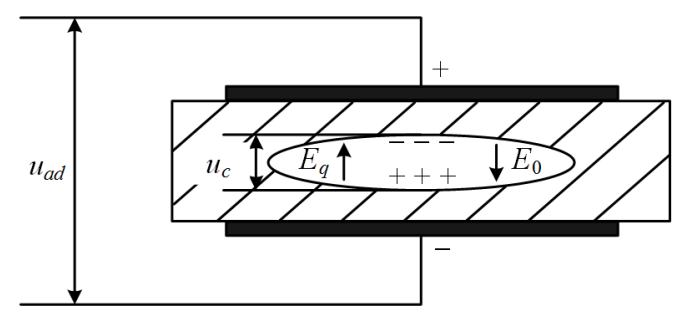

Fig. 3. Discharge distribution of air gap when discharge happened 


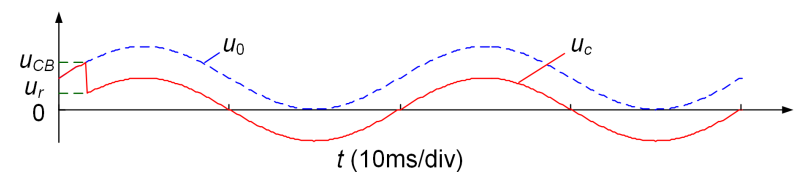

Fig. 4. Ideal voltage over the cavity under pulsating DC voltage with low amplitude

surface, as is shown in Fig. 4. The electrical field generated by the space charge keeps stable in absence of the volume resistivity and dielectric constant. $u_{c}$ is usually lower than the breakdown voltage $u_{C B}$ after the first PD and the discharges pause.

Considering the volume resistivity and dielectric constant of the insulation, the surface charge $q$ diffuses into the dielectric bulk due to conductivity of the cavity surface. The $u_{q}(t)$ can be approximated by:

$$
u_{q}(t)=-u_{q} e^{-t / \tau}
$$

where $u_{q}$ is the instantaneous amount of charge at the cavity surface of the previous PD. $\tau$ is the time constant of the charge relaxation process, which can be approximately expressed as [18]:

$$
\tau=\rho_{v} \varepsilon_{r}
$$

where $\rho_{v}$ is the volume resistivity of the insulation, and the value of the $\rho_{v}$ approximates $1 \times 10^{9} \Omega \cdot \mathrm{m} . \varepsilon_{r}$ is dielectric constant of the insulation, $\varepsilon_{r}=2.3 \varepsilon_{0} \approx 20.36 \times 10^{-12} \mathrm{~F} / \mathrm{m}$, and $\tau \approx$ $20.36 \mathrm{~ms}$.

The condition of recurrent PD is reported as follow:

$$
u_{c}(t)=u_{0}-u_{q}\left(1-e^{-t / \tau}\right) \geq u_{C B}
$$

Based on Eq. (1), Eq. (4) can be expressed as Eq. (5).

$$
\begin{aligned}
u_{c}(t) & =u_{a d}-u_{q}\left(1-e^{-t / \tau}\right) \geq u_{C B} \\
& =u_{a c}(1+\sin (\omega t+\varphi))-u_{q}\left(1-e^{-t / \tau}\right) \geq u_{C B}
\end{aligned}
$$

The PD repetition rate $N$ can also be calculated in this way. The time interval of the PDs can be expressed as Eq. (6).

$$
\Delta t=\tau \ln \left(\frac{u_{q}}{u_{q}+u_{C B} \quad u_{0}}\right)=\tau \ln \left(1 \frac{u_{C B} u_{0}}{u_{q}+u_{C B} \quad u_{0}}\right)
$$

Based on the Taylor's formula, Eq. (6) can be expressed as Eq. (7).

$$
\begin{aligned}
\Delta t & =\tau\left[\frac{u_{C B}-u_{0}}{u_{q}+u_{C B}-u_{0}}+\left(\frac{u_{C B}-u_{0}}{u_{q}+u_{C B}-u_{0}}\right)^{2}+\cdots\right] \\
& \approx \tau\left(\frac{u_{C B}-u_{0}}{u_{q}+u_{C B}-u_{0}}\right)
\end{aligned}
$$

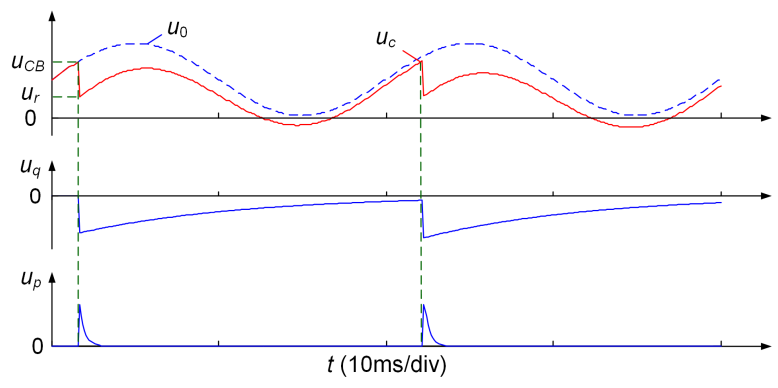

Fig. 5. Voltages variations of cavity discharge under pulsating DC voltage with low amplitude

The PD repetition rate $N$ is a maximum when $N=1 / \Delta t$. The formula of the cavity discharge repetition rate under pulsating DC voltage can be approximately written as:

$$
N=\frac{1}{\tau}\left(1+\frac{u_{q}}{u_{C B}-u_{0}}\right)
$$

Due to $u_{0} \propto u_{a d}$, the discharge recurrent rate is bigger than that under DC voltage [11]. The hazard of PD under pulsating DC voltage is more severe than that under DC voltage.

The voltage variations of $u_{0}, u_{c}, u_{q}(t), u_{p}(t)$ are illustrated in Fig. 5. At the inception pulsating DC voltage, the PD pulse signals appear in the phase range from $0^{\circ}$ to $90^{\circ}$ of the power frequency.

With the increase of the applied voltage, the voltages variations can be shown in Fig. 6 . When $u_{0}$ exceeds the breakdown voltage $u_{C B}$, PDs will happen in the cavity. The $u_{c}$ makes $u_{q}$ decrease and is lower than the breakdown voltage. PD is paused and the voltage $u_{q}(t)$ will decrease versus the time. $u_{c}$ will increase with the increase of the applied voltage until the $u_{c}$ reaches $u_{C B}$, and the PD will happen for a second time. The discharge process will repeat again and again following this rule. The previous surface discharge cannot leak out entirely due to the high volume resistivity of the oil-immerged paper. Thus, if the surface charges generated from different discharges are equaled and there are $n$ times of discharges in the positive power frequency phase, the voltage due to the surface charges equals to $-u_{q} \mathrm{e}^{-t 1 / \tau}-u_{q} \mathrm{e}^{-t 2 / \tau}-\ldots-u_{q} \mathrm{e}^{-t n / \tau}$. The voltage due to the surface charges is high enough for $u_{i}$ to reach $u_{C B}$ as shown in Fig. 6. PD pulse signals will occur again. However, the direction of the surface charge distribution is opposite and the surface charges will be neutralized. The absolute value of the $u_{q}(t)$ decreases and the $u_{c}$ will not be higher than $u_{C B}$ in the negative power frequency cycle after several times of discharges. Then PD will pause and then occur in the positive power frequency cycle again with variation of the applied voltage. Due to the leak of the surface charges, the discharge number in the negative power frequency cycle is smaller than that in the positive power frequency cycle.

Once the applied voltage reaches a big value and $u_{c}$ in 


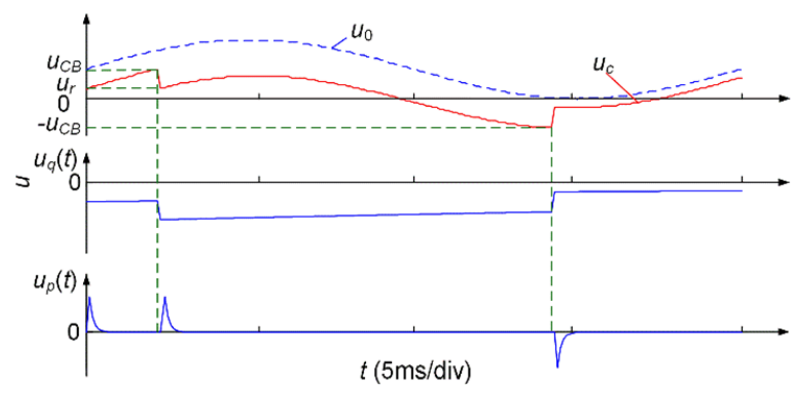

Fig. 6. Voltage variations of cavity discharge under pulsating DC voltage with higher amplitude

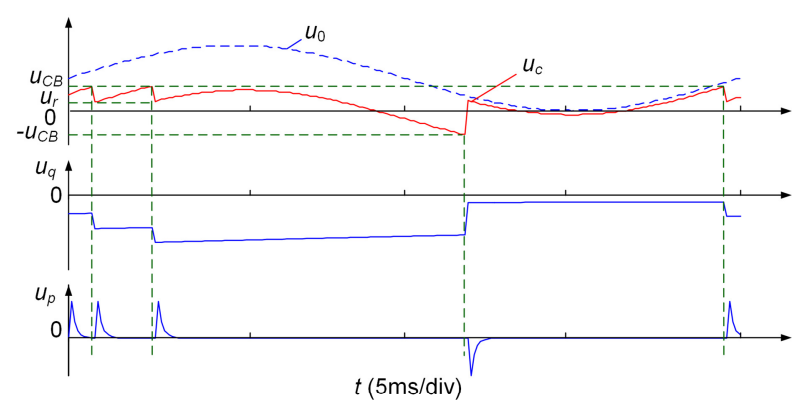

Fig. 7. Voltage variations of cavity discharge under pulsating DC voltage when the amplitude is high enough

the final phase domain of the power cycle is higher than $u_{C B}$, the PD pulse signals will occur in the phase domain. It is very different from the phase-dissolved distribution of oil-paper insulation under AC voltage. The discharge process can be seen in Fig. 7.

\subsection{Analyses of surface and floating discharges}

The surface discharge process can be divided into two stages, which are shown in Fig. 8. At the initiate stage of the surface discharge, electrical trees grow from the high voltage electrode to the ground electrode. In this stage, there are an electrode and oil-immerged paper between the discharge channels. The electrons and negative ions will dissipate quickly because of the electrical field and high conductivity of the copper electrodes. However, the positiveions will accumulate in the oil-immerged paper side. Due to the low applied voltage, the mechanism of surface discharge is similar with that of the cavity discharge.

With the increase of the applied voltage and the growing of the electrical trees, the discharge channels will reach the ground electrode and the ions can move more easily in the discharge channels. Under this condition, the electrodes are bridged by the discharge channels and the discharge voltage is relatively lower. The electrons leak out more quickly than the positive ions for the reason of the positive electrical field and smaller quality. An inverse voltage due to the positive ions will be produced between the discharge channels. The discharge intervals are so short that the

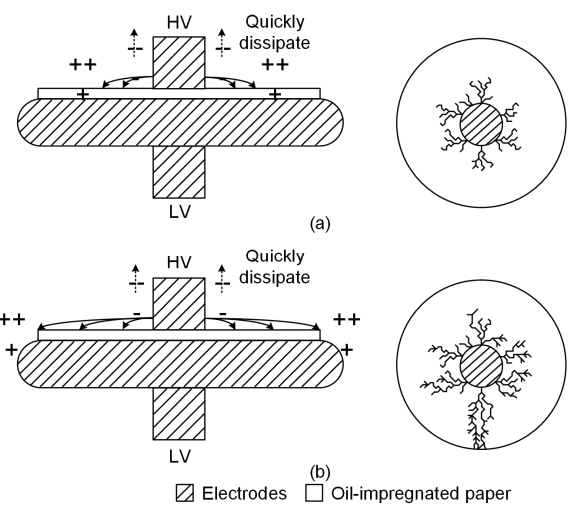

Fig.8. Discharge process of surface discharge

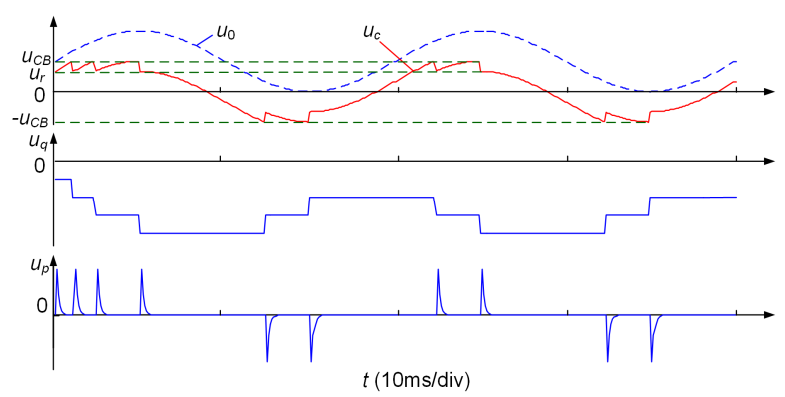

Fig. 9. Voltage variations of surface discharge when the discharge approaches are bridged.

positive ions can be considered constant. Once the $u_{c}$ is lower than $-u_{C B}$, the direction of discharge channel and space discharge are inversed. Under these hypotheses, the phase-dissolved distribution of surface discharge under pulsating DC voltage remains symmetric, which can be seen in Fig. 9.

When a particle floating in the oil-immerged paper, induced discharges will happen in the positive power frequency phases of the pulsating DC voltage. Under the applied voltage, discharge channels will grow around the electrode like the surface discharge. Besides, some discharge channels will be generated around the floating particle, thus the floating discharge will develop to surface discharge.

\subsection{Theoretical analyses of corona discharges}

The mechanism of corona discharge under AC voltage is quite explicit, which can be seen in [19]. There is also polarity effect for corona discharge under pulsating DC voltage. Under the positive voltage, the electrons will be readily diffused into the anode, leaving the positive space charge. The space charge will cause a reduction in the field strength closing to the anode and will increase the field farther away from it at the same time, which is illustrated in Fig. 10(a). With the negative point, the positive space charge will remain in the space between the negative charge and the point. The field around the point is grossly enhanced, but the field in the ionization region is 


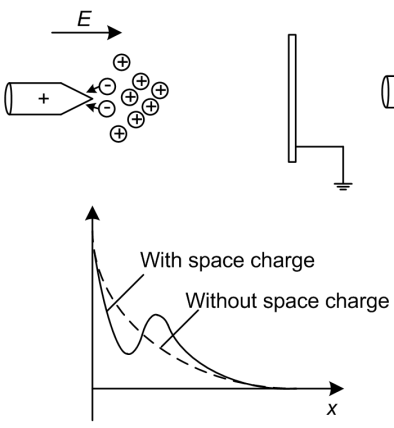

(a) Positive corona

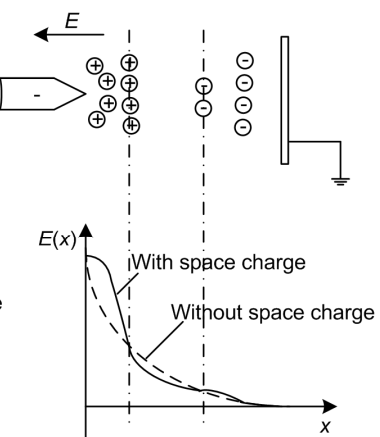

(b) Negative corona
Fig. 10. Space charge built-up in point-plane gap and field variations under the applied voltage with two polarities [20]

drastically reduced. The field variation is illustrated in Fig. 10 (b). It is clear that the negative inception voltage is lower than the positive inception voltage.

Under the pulsating DC voltage, discharges mainly occur in the region with high voltage amplitude. When the applied voltage reaches almost zero, the electrical field due to the space charge will be higher than the external electrical field. Some discharges in opposite directions will happen in the zero regions of the applied voltage for both positive and negative corona discharges.

\section{PD experiments under Pulsating DC Voltage}

\subsection{Artificial insulation defect models}

Four types of artificial insulation defect models [21], as shown in Fig. 11, were designed for experiments to generate PD signals. Fig. 11(a) shows the model of air cavity discharge with three layers of oil-impregnated papers and a sphere-to-board electrode. Fig. 11(b) shows a model to generate surface discharge in oil with a cylinderto-board electrode used. Fig. 11(c) is the model of floating

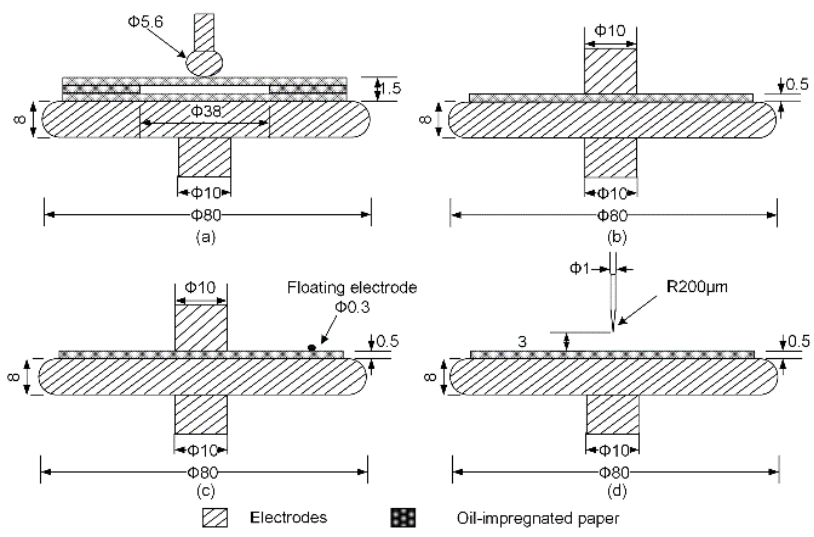

Fig. 11. Four types of artificial defect models: (a) air cavity discharge; (b) surface discharge; (c) floating discharge; (d) corona discharge. discharge. A metal particle is fixed on the oil-impregnated paper and a cylinder-to-board electrode system is used. Fig. 11(d) presents a corona discharge model with a needle-toplate electrode system. All models were immersed in oil to simulate oil-paper insulation defects.

\subsection{Setup and conditions of PD experiment}

The experimental setup of PD detection under pulsating DC voltage is shown in Fig. 12. The high voltage was detected by a high voltage probe manufactured by Tektronix ${ }^{\circledR}$. The artificial defect models were put into a synthetic glass box filled with transformer oil and the experiments were carried out in an electromagnetic shielded laboratory. A Rogowski coil sensor, $S$ in Fig. 12, was used for PD detection in the experiments. The sensor has good performance with a wide frequency pass-band from $50 \mathrm{kHz}$ to $10 \mathrm{MHz}$. A high performance digital oscilloscope with a sampling frequency of $50 \mathrm{MS} / \mathrm{s}$ was adopted to observe and record PD signals obtained by the sensor.

Table 2 reveals the inception voltages and test voltages of the four PD models from experiments. 3000 cycle numbers of PD signals generated from each defect model were detected for establishing statistical histograms when discharge sufficiently. 25 samples were obtained at each

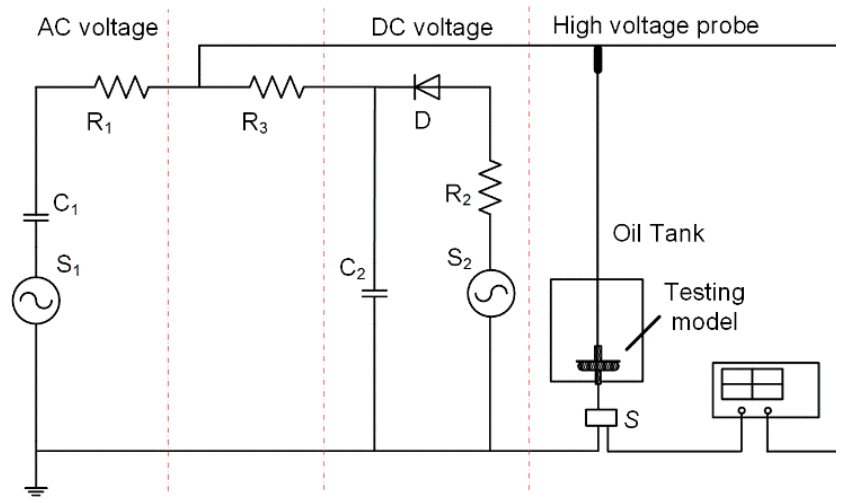

Fig.12.Setup of PD experiment in laboratory

Table 2. PD experiment conditions

\begin{tabular}{|c|c|c|c|}
\hline PD model & $\begin{array}{c}\text { Inception voltage } \\
(\mathrm{kV})\end{array}$ & $\begin{array}{c}\text { Test voltage } \\
(\mathrm{kV})\end{array}$ & $\begin{array}{l}\text { Sample } \\
\text { numbers }\end{array}$ \\
\hline \multirow{3}{*}{ Cavity } & \multirow{3}{*}{3.8} & 4 & 25 \\
\hline & & 7 & 25 \\
\hline & & 10 & 25 \\
\hline \multirow{3}{*}{ Surface } & \multirow{3}{*}{12.8} & 13 & 25 \\
\hline & & 14 & 25 \\
\hline & & 15 & 25 \\
\hline \multirow{3}{*}{ Floating } & \multirow{3}{*}{10.8} & 11 & 25 \\
\hline & & 12 & 25 \\
\hline & & 13 & 25 \\
\hline \multirow{2}{*}{ Corona $(+)$} & \multirow{2}{*}{14.4} & 17 & 25 \\
\hline & & 21 & 25 \\
\hline \multirow{2}{*}{ Corona (-) } & \multirow{2}{*}{-12.8} & -16 & 25 \\
\hline & & -20 & 25 \\
\hline
\end{tabular}



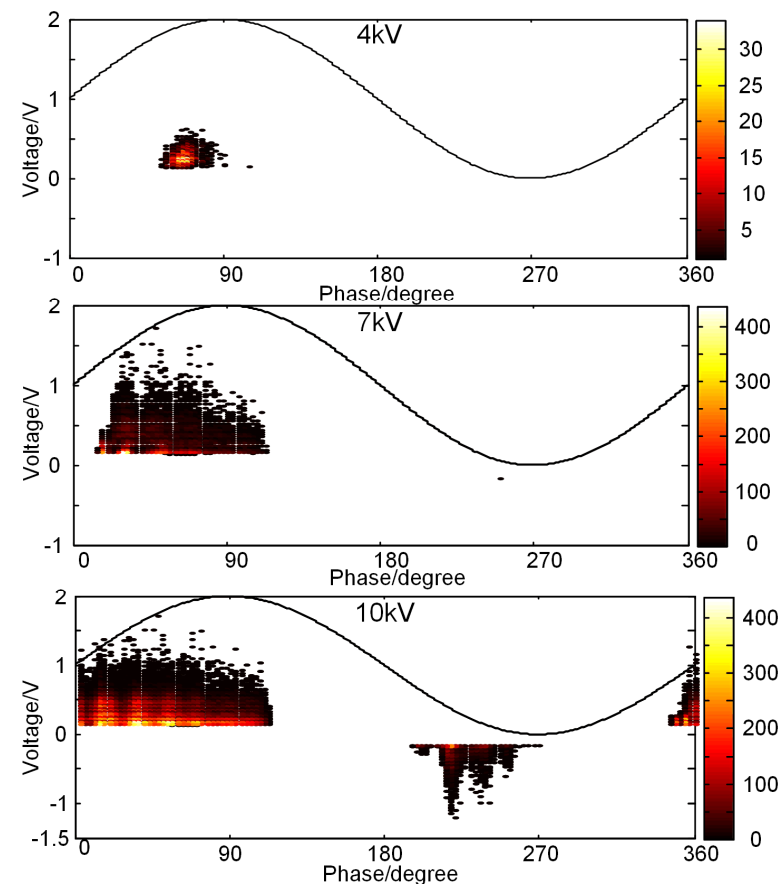

Fig. 13. PD phase-resolved distributions of cavity discharge.
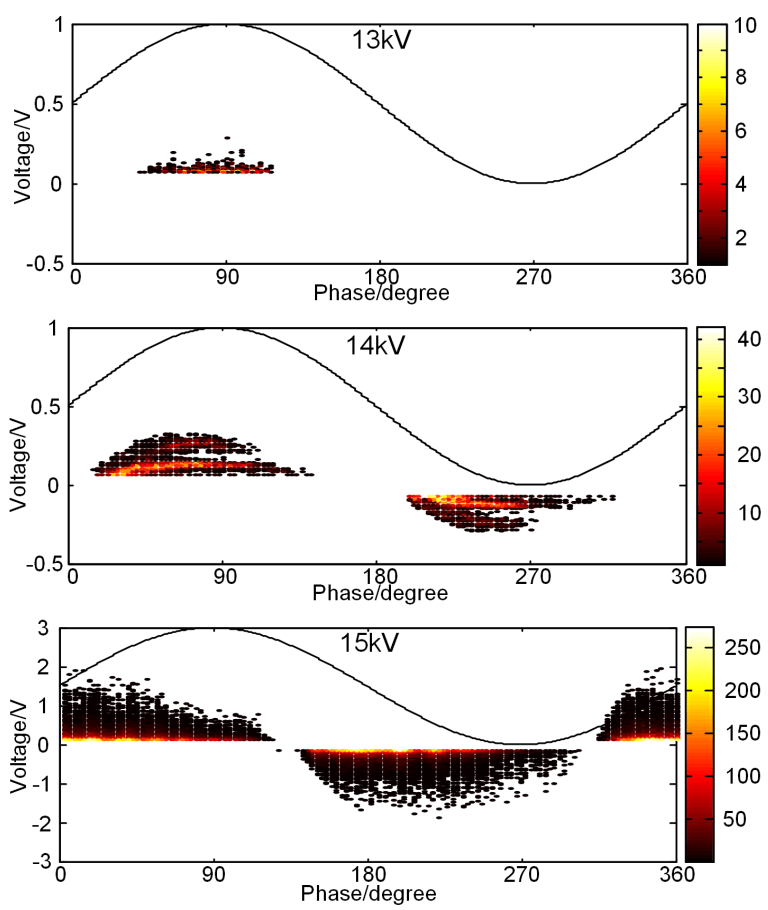

Fig. 14. PD phase-resolved distributions of surface discharge.

voltage for every PD model. The total number of sample data of PD signals was 200.

\subsection{Results and analysis}

Fig. 13 shows the phase-resolved distributions generated from the cavity model under pulsating DC voltage with different amplitudes. PD impulse signals first occurred in
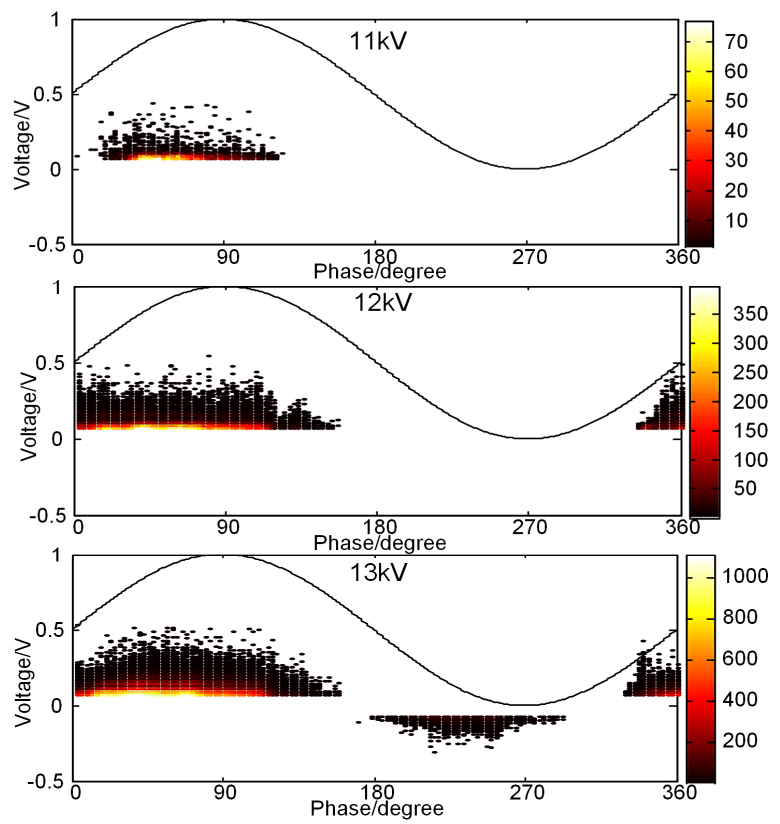

Fig. 15. PD phase-resolved distributions of floating discharge.

the positive half cycle of the power frequency period. PD pulses also occurred in wider phases with an increase in the test voltages. When the applied voltage was $4 \mathrm{kV}$, the PD number in 3000 cycle was 2491, that is, PD repetition rate is 41.52 . If the thickness of cavity is $0.5 \mathrm{~mm}, u_{C B} \approx 3$ $\mathrm{kV}$ can be obtained according to Eq. (24) in [22], and $u_{r}=0.2 u_{C B}, u_{q}=2.4 \mathrm{kV}, u_{0}=4 \mathrm{kV}$. The $\mathrm{PD}$ repetition rate $N=44.45$ can be calculated by Eqs. (3) and (8), which was consistent with the theoretical analysis. The phase-resolved distributions of the positive and negative half cycles turned out to be asymmetrical.

Fig. 14 indicates the variations in phase-resolved distributions of the surface model under pulsating DC voltage. First, PD pulses occurred in the positive half cycle of the power frequency period, but they were wider than those generated from cavity discharge. As the applied voltages increasing, PD pulse signals would appear in the wider phase, as shown in Fig. 14. The phase-resolved distributions of the positive and negative half cycles show certain similarities. The discharge process is also according the theoretical analysis.

Fig. 15 shows the variations in phase-resolved distributions generated from the floating model under pulsating DC voltage with different amplitudes. PD pulses mainly occurred in the positive half cycle of the power frequency period and would occur at $300^{\circ}$ to $360^{\circ}$ when the applied voltage was large enough. With the increase of the applied voltage, the floating discharge would develop to surface discharge easily. This phenomenon is shown in the third figure of Fig. 15.

Fig. 16 shows the phase-resolved distributions constructed from the positive corona model under pulsating DC voltage. PD pulses first appeared in the positive half 

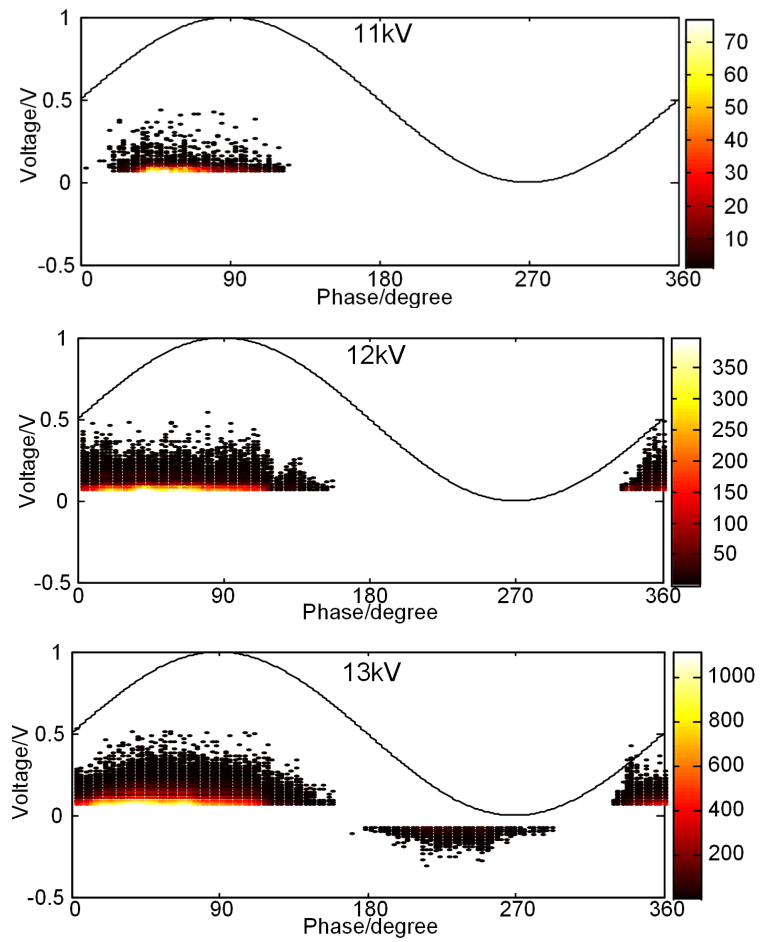

Fig. 16. PD phase-resolved distributions of positive corona discharge.

cycle of the power frequency period. As the test voltages increased, the phase-resolved distribution of the PD impulse signals became wider, and the amplitudes and discharge numbers were larger. PDs would occur in the phase range around $270^{\circ}$ of power frequency when the applied voltage was high enough.

Fig. 17 shows the phase-resolved distributions of negative corona discharge under pulsating DC voltages with different amplitudes. PD pulses first occurred in the negative half cycle of the power frequency period. The amplitudes of the impulse signals were small. With an increase in the applied voltages, the PD phase-resolved distribution in the negative half cycle became wider, and the amplitudes and discharge numbers were larger. PD pulse signals also appeared in the positive half cycle of the power frequency period. However, the phase-resolved distribution was narrower, the amplitudes were larger, and the discharge number was less than the PD impulse signals in the negative half cycle.

Experimental results reveal that the PD processes of the different discharge models are in agreement with the theoretical analysis. The PD phase-resolved distributions under pulsating DC voltage are different from those under individual AC or DC voltage, which can be seen in [23]. For the four PD defect models under pulsating DC voltages, PD pulses first appeared at the maximum value of the applied voltage, and as the applied voltage increased, the resolved PD phase distribution became wider. The phaseresolved distributions of the positive and negative half
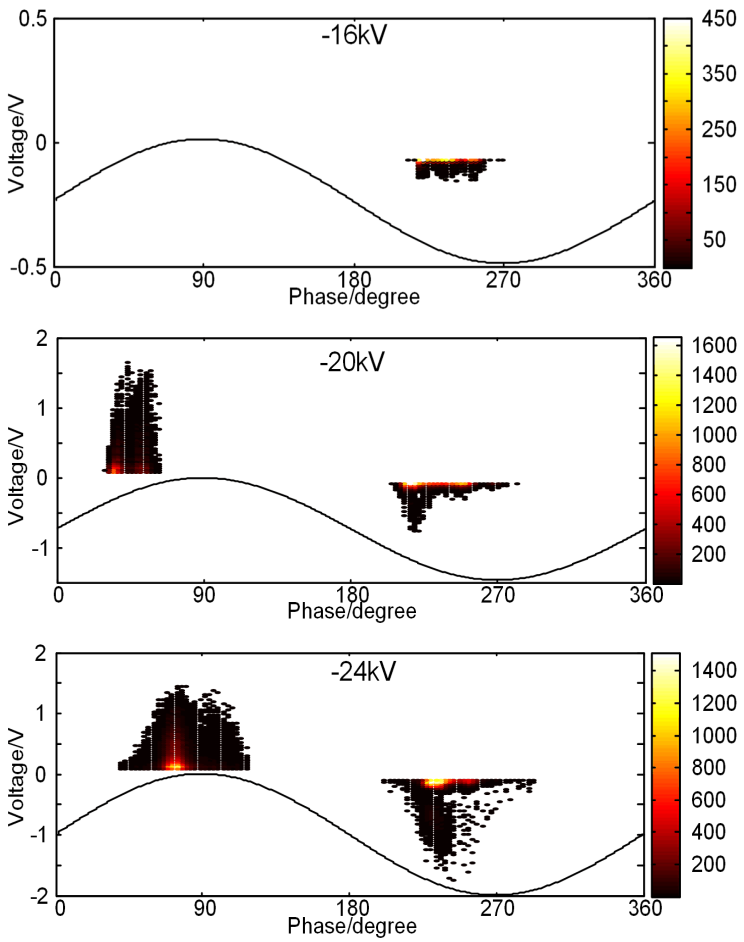

Fig.17. PD phase-resolved distributions of negative corona discharge.

cycles were asymmetrical.

When sufficiently discharged, the PD phase-resolved distributions generated from the different discharge models are different from each other. The PD pulses generated from cavity discharge model mainly concentrate in positive half cycle of the power frequency period; PD pulses would appear in the negative half-cycle only when the test voltage was high enough. The PD phase-resolved distributions of the surface model were wider than those of the cavity discharge, and the distributions of the positive and negative half cycles were approximately symmetrical. The floating discharge pulses mainly occurred in the positive half cycle and could develop to surface discharge easily. The phase-resolved distribution of corona discharge was narrower than those of other discharge models, and there is a polarity effect for corona discharge under pulsating DC voltage. These difference in phase-resolved distributions are useful in further studies for pattern recognition of PDs in converter transformers.

\section{Conclusion}

This paper presents partial discharge properties of oilpaper insulation under pulsating DC voltage. Theoretical PD mechanisms of different insulation defected models under pulsating DC voltage were proposed. For confirmation of the proposed theories, PD experiments under pulsating DC voltage were done. The results of the work 
can be summarized as follows:

(1) Theoretical PD mechanisms of different insulation defected models under pulsating DC voltage showed that PD pulses first appeared at the maximum value of the applied voltage and the phase-resolved distributions of the positive and negative half cycles were asymmetrical, which were different from those under individual AC or DC voltage.

(2) Four models were developed to simulate typical insulation defects in converter transformers and PD signals were detected. The phase-resolved distributions of PDs generated from the four models were in agreement with the theoretical analysis but different from each other.

(3) PD pulses generated from cavity model, floating model and positive corona model mainly concentrate in positive half cycle, but the negative corona discharge model is opposite. The phase-dissolved distributions of surface discharge were approximately symmetrical.

There is still work to be done for pattern recognition of PDs under pulsating DC voltage. In addition, PD mechanism, experiments, and pattern recognition in converter transformer will be further investigated.

\section{Acknowledgements}

The authors acknowledge National Basic Research Program of China (973 Program, No. 2012CB215200), Natural Science Foundation of China (51177180, 51321063) and for supporting this study. We also thank the support and funding of the 111 Project from the Ministry of Education, China (B08036).

\section{References}

[1] L. Luo, Y. Li, J. Xu, el ta. "A New Converter Transformer and a Corresponding Inductive Filtering Method for HVDC Transmission System", IEEE Trans. Power Del., Vol. 23, pp. 1426-1431, 2008.

[2] J. Li, Y. Wang, L. Bao, "Two Factors Failure Model of Oil-Paper Insulation Aging under Electrical and Thermal Multi-stress", Journal of Electrical Engineering \&Technology, Vol. 9, pp. 957-963, 2014.

[3] Y. Wang, J. Li, S. Wu, P. Sun, "Influence of Electrical Aging on Space Charge Dynamics of Oil-Impregnated Paper Insulation under AC-DC Combined Voltages", Journal of Electrical Engineering \& Technology, Vol. 8, pp. 1512-1519, 2013.

[4] J. Li, Y. Wang, L. Bao, "Space Charge Behavior of Oil-Impregnated Paper Insulation Aging at AC-DC Combined Voltages", Journal of Electrical Engineering \&Technology, Vol. 9, pp. 635-642, 2014.
[5] D. J. Swaffield, P. L. Lewin, G. Chen, el ta. "Partial discharge characterization of streamers in liquid nitrogen under applied AC voltages", IEEE Trans. Dielectr. Electr. Insul., Vol. 15, pp. 635-646, 2008.

[6] S. Senthil Kumar, "PD data analysis and evaluation of partial discharge patterns for uniform characterization", IEE Proceedings Science, Measurement and Technology, Vol. 151, pp. 278-284, 2004.

[7] J. Li, R. Liao, S. Grzybowski, el ta. “Oil-paper aging evaluation by fuzzy clustering and factor analysis to statistical parameters of partial discharges", IEEE Trans. Dielectr. Electr. Insul., Vol. 17, pp. 756-763, 2010.

[8] J. Li, C. Sun, S. Grzybowski, el ta. "Partial discharge image recognition using a new group of features", IEEE Trans. Dielectr. Electr. Insul., Vol. 13, pp. 12451253, 2006.

[9] J. Li, C. Sun, S. Grzybowski, "Partial Discharge Image Recognition Influenced by Fractal Image Compression", IEEE Trans. Dielectr. Electr. Insul., Vol. 15, pp. 496-504, 2008.

[10] U. Fromm, "Interpretation of partial discharges at dc voltages", IEEE Trans. Dielectr. Electr. Insul., Vol. 2, pp. 761-770, 1995.

[11] P. H. F. Morshuis, J. J. Smit, "Partial discharges at DC voltage: their mechanism, detection and analysis", IEEE Trans. Dielectr. Electr. Insul., Vol. 12, pp. 328340, 2005.

[12] W. Si, J. Li, P. Yuan, el ta. "Digital detection, grouping and classification of partial discharge signals at DC voltage", IEEE Trans. Dielectr. Electr. Insul., Vol. 15, pp. 1663-1674, 2008.

[13] R.Sarathi, G.Koperundevi, "Investigation of partial discharge activity of single conducting particle in transformer oil under DC voltages using UHF technique", IET Science, Measurement \& Technology, Vol. 3, pp. 325-333, 2009.

[14] R. Sarathi, A. V. Giridhar, A. Mani, el ta. "Investigation of partial discharge activity of conducting particles in liquid nitrogen under DC voltages using uhf technique", IEEE Trans. Dielectr. Electr. Insul., Vol. 15, pp. 655-662, 2008.

[15] T. Do, O. Lesaint, J. L. Auge, "Streamers and partial discharge mechanisms in silicone gel under impulse and AC voltages", IEEE Trans. Dielectr. Electr. Insul., Vol. 15, pp. 1526-1534, 2008.

[16] A. J. Vandermaar, M. Wang, J. B. Neilson, el ta. "The electrical breakdown characteristics of oilpaper insulation under steep front impulse voltages", IEEE Trans. Power Del.,Vol. 8, pp. 1926-1935, 1994.

[17] Y. Kamata, K. Endoh, S. Furukawa, el ta. "Dielectric strength of oil-immersed transformer insulation with superimposed AC and lightning impulse voltage", IEEE Trans. Dielectr. Electr. Insul., Vol. 25, pp. 683687, 1990.

[18] E. Kuffel, W.S. Zaengl, J. Kuffel. High Voltage Engi- 
neering: Fundamentals, Butterworth-Heinemann in Oxford, 2000.

[19] Г.С. Kuschynski, Partial discharge of high voltage electrical equipment, China Water Power Press, Beijing, 1984. (in Chinese)

[20] J. Li, Z. Zhang, P. Zou, el ta. "Lightning impulse breakdown characteristics and electro dynamic process of insulating vegetable oil-based nanofluid", Mod. Phys. Lett. B, vol. 26, p. 1250095.

[21] J. Li, T. Jiang, Robert F. Harrison, el ta. "Recognition of Ultra High Frequency Partial Discharge Signals Using Multi-scale Features", IEEE Trans. Dielectr. Electr. Insul., vol. 19, pp. 1412-1419, 2012.

[22] L. Niemeyer. A Generalized Approach to Partial Discharge Modeling", IEEE Trans. Dielectr. Electr. Insul., 1995, vol. 2, 510-527, 1995.

[23] F. H. Kreuger, Partial discharge detection in highvoltage equipment, Butterworths in London, Boston, 1989.

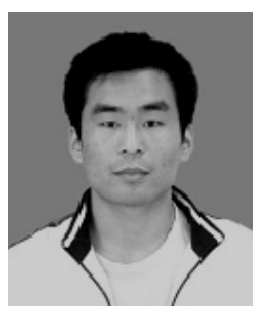

Lianwei Bao received the Bachelor degree in electrical engineering in 2010 , from Southwest Jiaotong University, Chengdu, China. He is currently a Ph.D. degree candidate in the electrical engineering college of Chongqing University, Chongqing, China. His major research interests include online condition monitoring and fault diagnosis of high voltage equipment, aging properties of insulation materials, and probabilistic analysis to insulation failure data.

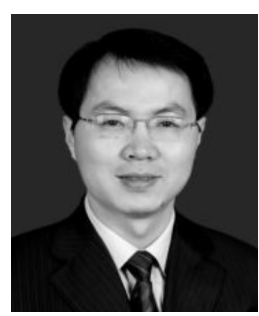

Jian Li received the M.S. and Ph.D degree in electrical engineering in 1997 and 2001, from Chongqing University, Chongqing, China. He is currently a professor and the Associate Dean of School of Electrical Engineering at Chongqing University. His major research interests include online condition monitoring and fault diagnosis of $\mathrm{HV}$ equipment, environment-friendly insulation materials and nano dielectrics. He is an author and co-author of more than 80 journal papers and 60 papers published in proceedings of international conferences.

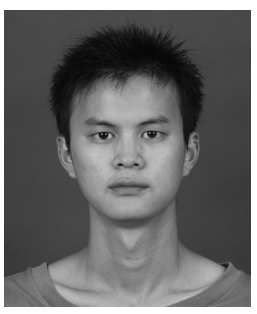

Jing Zhang received the Bachelor degree in electrical engineering and automation in 2012, from China University of Mining and Technology, Xuzhou, China. He is currently a Ph.D. candidate at Chongqing University. His major research interests include online condition monitoring and fault diagnosis of high voltage equipment, aging properties and diagnosis of insulation materials and structures in transformers.

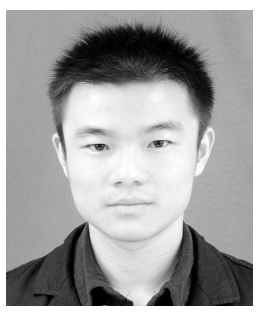

TianYan Jiang received the Bachelor degree and Ph.D degree in electrical engineering in 2008 and 2013, from Chongqing University, Chongqing, China. He is currently a teacher at Chongqing University of Technology. His major research interests include partial discharges, online detection and insulation fault diagnosis of electrical equipment.

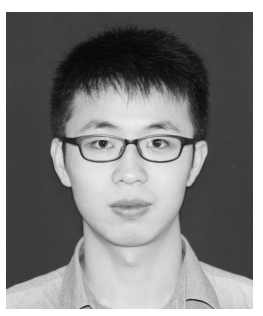

Xudong Li received the Bachelor degree in electrical engineering in 2013, from Chongqing University, Chongqing, China. He is currently a $\mathrm{Ph} . \mathrm{D}$. degree candidate in the electrical engineering college of Chongqing University, Chongqing, China, His major research interests include partial discharge, new sensors for condition monitoring and insulation fault diagnosis of high voltage equipment. 